Supplement of Proc. IAHS, 383, 25-30, 2020

https://doi.org/10.5194/piahs-383-25-2020-supplement

(C) Author(s) 2020. This work is distributed under

the Creative Commons Attribution 4.0 License.

(c) (i)

Supplement of

\title{
Analysis of water level variations in the Huangzhuang reach (Hanjiang River, China) during recent years
}

Xiao Xiao et al.

Correspondence to: Bao Qian (jacber@163.com)

The copyright of individual parts of the supplement might differ from the CC BY 4.0 License. 
Fig 2 Relationsship between annual average daily water levels and corresponding discharge at the Huangzhuang station for the period 2000-2017

\begin{tabular}{|c|r|c|c|c|}
\hline Year & $\mathrm{Zmax}(\mathrm{m})$ & $\mathrm{Q}(\mathrm{m} 3 / \mathrm{s})$ & $\mathrm{Zmin}(\mathrm{m})$ & $\mathrm{Q}(\mathrm{m} 3 / \mathrm{s})$ \\
\hline 2000 & 45,78 & 8340 & 39,96 & 189 \\
\hline 2001 & 43,09 & 2020 & 41,12 & 688 \\
\hline 2002 & 43,82 & 2920 & 40,51 & 381 \\
\hline 2003 & 47,48 & 14600 & 40,4 & 339 \\
\hline 2004 & 44,57 & 4620 & 40,99 & 830 \\
\hline 2005 & 47,36 & 16900 & 41,26 & 920 \\
\hline 2006 & 43,26 & 2550 & 40,29 & 535 \\
\hline 2007 & 45,8 & 8410 & 40,04 & 425 \\
\hline 2008 & 45,15 & 7580 & 40,02 & 340 \\
\hline 2009 & 44,01 & 4460 & 40,45 & 623 \\
\hline 2010 & 47,15 & 13500 & 40,48 & 720 \\
\hline 2011 & 47,35 & 13900 & 39,9 & 451 \\
\hline 2012 & 44,1 & 4970 & 40,17 & 540 \\
\hline 2013 & 42,51 & 2030 & 40,09 & 370 \\
\hline 2014 & 43,15 & 2400 & 39,73 & 295 \\
\hline 2015 & 43,75 & 2910 & 39,98 & 503 \\
\hline 2016 & 43,28 & 2380 & 39,94 & 501 \\
\hline 2017 & 48,62 & 13700 & 39,97 & 507 \\
\hline
\end{tabular}


Fig 3 Surface water fall in different reaches of the Hanjiang River for different discharge ranges and time periods in 2005-2017

\begin{tabular}{|c|c|c|c|c|}
\hline \multicolumn{5}{|c|}{ (a) Zhuandouwan ${ }^{\sim}$ Huangzhuang reach } \\
\hline time interval & Under $1000 \mathrm{~m}^{3} / \mathrm{s}$ & $1000 \sim 500$ & $5000 \sim 100$ & Above $10000 \mathrm{~m}^{3} / \mathrm{s}$ \\
\hline $2005 \sim 2008$ & 4,77 & 4,83 & 4,95 & 4,85 \\
\hline 2009 2013 & 4,84 & 4,88 & 4,98 & 4,77 \\
\hline $2014 \sim 2015$ & 4,36 & 4,35 & & \\
\hline 2016 2017 & 4,31 & 4,12 & 4,07 & 3,88 \\
\hline
\end{tabular}

\begin{tabular}{|c|c|c|c|c|}
\hline \multicolumn{5}{|c|}{ (b) Huangzhuang Datong reach } \\
\hline time interval & Under $1000 \mathrm{~m}^{3} / \mathrm{s}$ & $\begin{array}{c}1000 \sim 500 \\
0 \mathrm{~m}^{3} / \mathrm{s}\end{array}$ & $\begin{array}{c}5000 \sim 100 \\
00 \mathrm{~m}^{3} / \mathrm{s}\end{array}$ & Above $10000 \mathrm{~m}^{3} / \mathrm{s}$ \\
\hline $2005 \sim 2008$ & 3,13 & 3,02 & 2,72 & 2,38 \\
\hline 2009 2013 & 2,84 & 2,82 & 2,49 & 2,22 \\
\hline $2014 \sim 2015$ & 2,01 & 2,57 & & \\
\hline $2016 \sim 2017$ & 1,78 & 2,01 & 3,01 & 3,27 \\
\hline \multicolumn{5}{|c|}{ (c) Datong Shayang reach } \\
\hline time interval & Under $1000 \mathrm{~m}^{3} / \mathrm{s}$ & $\begin{array}{c}1000 \sim 500 \\
0 \mathrm{~m}^{3} / \mathrm{s}\end{array}$ & $\begin{array}{c}5000 \sim 100 \\
00 \mathrm{~m}^{3} / \mathrm{s}\end{array}$ & Above $10000 \mathrm{~m}^{3} / \mathrm{s}$ \\
\hline $2005 \sim 2008$ & 3,63 & 3,61 & 3,31 & 3,17 \\
\hline 2009 2013 & 3,16 & 3,37 & 3,32 & 3,16 \\
\hline $2014 \sim 2015$ & 0,63 & 1,58 & & \\
\hline $2016 \sim 2017$ & 0,84 & 1,69 & 3,25 & 3,14 \\
\hline
\end{tabular}


Fig4 The cross section at the bridge in the Hanjiang River

\begin{tabular}{|c|c|c|c|}
\hline 2016 & & \begin{tabular}{|r|}
2012 \\
\end{tabular} & \\
\hline distance & elevation & distance & elevation \\
\hline 0 & 50 & 0 & 49,9 \\
\hline 33,71708 & 43 & 26,17529 & 44,1 \\
\hline 58,80697 & 43 & 92,11657 & 44 \\
\hline 134,6988 & 44 & 263,9244 & 45 \\
\hline 186,2724 & 44,9 & 505,9986 & 45,6 \\
\hline 335,497 & 45 & 802,4054 & 45,5 \\
\hline 467,1799 & 45,2 & 1008,964 & 45,6 \\
\hline 663,2656 & 45,7 & 1132,846 & 45,1 \\
\hline 844,1602 & 45,5 & 1243,699 & 46 \\
\hline 998,04 & 48,3 & 1395,168 & 46 \\
\hline 1033,511 & 46 & 1517,209 & 45 \\
\hline 1130,874 & 45,5 & 1553,103 & 44 \\
\hline 1297,667 & 45,5 & 1622,821 & 42 \\
\hline 1486,847 & 45 & 1646,571 & 42 \\
\hline 1528,545 & 44 & 1679,444 & 42,29 \\
\hline 1588,499 & 43 & 1683,263 & 39,18 \\
\hline 1675,54 & 42 & 1685,778 & 39 \\
\hline 1685,417 & 39 & 1689,348 & 38 \\
\hline 1690,214 & 39 & 1697,269 & 37 \\
\hline 1698,318 & 39 & 1708,012 & 36 \\
\hline 1704,112 & 39 & 1730,59 & 35 \\
\hline 1718,455 & 39 & 1735,474 & 35 \\
\hline 1749,88 & 39 & 1780,72 & 36 \\
\hline 1783,232 & 39 & 1807,548 & 37 \\
\hline 1813,317 & 39 & 1845,729 & 38 \\
\hline 1834,661 & 39 & 1868,068 & 38 \\
\hline 1941,036 & 38,1 & 1899,382 & 37 \\
\hline 1945,981 & 38 & 1920,728 & 36 \\
\hline 1951,357 & 37 & 1937,799 & 35 \\
\hline 1959,601 & 36 & 1945,52 & 34 \\
\hline 1967,136 & 35 & 1952,985 & 33 \\
\hline 2018,313 & 35 & 1965,778 & 33 \\
\hline 2040,235 & 36 & 1975 & 34 \\
\hline 2062,478 & 37 & 1984,378 & 35 \\
\hline 2101,361 & 37 & 1997,934 & 36 \\
\hline 2111,084 & 36 & 2010,364 & 37 \\
\hline 2120,019 & 35 & 2022,342 & 38 \\
\hline 2133,082 & 34 & 2069,769 & 38,9 \\
\hline 2140,541 & 33 & 2085,915 & 38 \\
\hline 2150,744 & 32 & 2100,886 & 37 \\
\hline 2158,266 & 31 & 2113,915 & 36 \\
\hline
\end{tabular}




\begin{tabular}{|r|r|l|r|r|}
\hline 2166,146 & 30 & & 2121,906 & 35 \\
\hline 2181,532 & 30 & & 2129,862 & 34 \\
\hline 2196,795 & 31 & & 2137,436 & 33 \\
\hline 2210,376 & 32 & & 2143,959 & 32 \\
\hline 2214,032 & 33 & & 2159,107 & 31 \\
\hline 2217,74 & 34 & & 2170,533 & 30 \\
\hline 2221,063 & 35 & & 2180,717 & 29 \\
\hline 2223,373 & 36 & & 2198,875 & 29 \\
\hline 2225,761 & 37 & & 2207,263 & 30 \\
\hline 2227,127 & 38 & & 2220,438 & 31 \\
\hline 2229,296 & 38,2 & & 2231,22 & 32 \\
\hline 2233,969 & 45,32 & & 2238,569 & 33 \\
\hline 2271,372 & 43 & & 2243,553 & 34 \\
\hline 2298,455 & 44 & & 2247,981 & 35 \\
\hline 2358,188 & 45 & & 2250,365 & 36 \\
\hline 2371,886 & 50 & & 2254,527 & 37 \\
\hline & & & 2257,321 & 38 \\
\hline & & & 2259,482 & 39 \\
\hline & & & 2261,759 & 38,9 \\
\hline & & & 2274,206 & 44,1 \\
\hline & & & 2304,287 & 44,1 \\
\hline & & & 2349,667 & 45 \\
\hline & & & 2371,886 & 50 \\
\hline
\end{tabular}


Fig5 Impact of construction work in 2016 on the stagedischarge relationship at the Road NO.2 road bridge over of the Hanjiang River in Zhongxiang

\begin{tabular}{|c|c|c|c|}
\hline 2016 & & 2012 & \\
\hline Water level(m) & Flow $\left(\mathrm{m}^{\mathrm{j}} / \mathrm{s}\right)$ & Water & Flow $\left(\mathrm{m}^{\mathrm{J}} / \mathrm{s}\right)$ \\
\hline 30,5 & 1,13395431 & 29,5 & 1,267754 \\
\hline 31 & 4,24205552 & 30 & 4,510693 \\
\hline 31,5 & 9,72309949 & 30,5 & 9,737654 \\
\hline 32 & 17,8426995 & 31 & 17,68249 \\
\hline 32,5 & 29,5492319 & 31,5 & 28,68484 \\
\hline 33 & 44,2146853 & 32 & 43,32965 \\
\hline 33,5 & 62,4700092 & 32,5 & 63,80891 \\
\hline 34 & 83,8413587 & 33 & 88,12488 \\
\hline 34,5 & 106,8281 & 33,5 & 108,5483 \\
\hline 35 & 133,431725 & 34 & 141,1119 \\
\hline 35,5 & 137,127799 & 34,5 & 179,4488 \\
\hline 36 & 179,939261 & 35 & 224,0425 \\
\hline 36,5 & 230,198775 & 35,5 & 246,9226 \\
\hline 37 & 289,126652 & 36 & 292,608 \\
\hline 37,5 & 426,195318 & 36,5 & 361,3571 \\
\hline 38 & 658,695004 & 37 & 445,5112 \\
\hline 38,5 & 792,488025 & 37,5 & 658,2811 \\
\hline 39 & 973,182391 & 38 & 961,2993 \\
\hline 39,5 & 1150,89578 & 38,5 & 1229,705 \\
\hline 40 & 1512,1985 & 39 & 1607,313 \\
\hline 40,5 & 1960,50449 & 39,5 & 2132,452 \\
\hline 41 & 2565,72056 & 40 & 2610,778 \\
\hline 41,5 & 3332,88411 & 40,5 & 3204,519 \\
\hline 42 & 4308,64246 & 41 & 4014,608 \\
\hline 42,5 & 5403,82795 & 41,5 & 5032,332 \\
\hline 43 & 6419,34752 & 42 & 6315,424 \\
\hline 43,5 & 7283,18403 & 42,5 & 7539,992 \\
\hline 44 & 8476,49409 & 43 & 9019,406 \\
\hline 44,5 & 10786,2383 & 43,5 & 10846,31 \\
\hline 45 & 12016,2554 & 44 & 13062,44 \\
\hline 45,5 & 14243,82147 & 44,5 & 14812,772 \\
\hline 46 & 17969,71219 & 45 & 16338,304 \\
\hline 46,5 & 21376,02134 & 45,5 & 18439,404 \\
\hline 47 & 23582,7585 & 46 & 21318,373 \\
\hline 47,5 & 28298,60851 & 46,5 & 24284,782 \\
\hline 48 & 33379,10459 & 47 & 26794,453 \\
\hline 48,5 & 38965,34001 & 47,5 & 32128,9 \\
\hline 49 & 45135,785 & 48 & 37845,977 \\
\hline \multirow[t]{3}{*}{49,5} & 51665,7595 & 48,5 & 43933,818 \\
\hline & & 49 & 50381,985 \\
\hline & & 49,5 & 57181,196 \\
\hline
\end{tabular}

